\title{
Molecular modification of coumarin dyes for more efficient dye sensitized solar cells
}

Cite as: J. Chem. Phys. 136, 194702 (2012); https://doi.org/10.1063/1.4711049

Submitted: 01 February 2012 . Accepted: 08 April 2012 . Published Online: 16 May 2012

Rocío Sánchez-de-Armas, Miguel A. San-Miguel, Jaime Oviedo, and Javier Fdez. Sanz

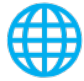

\section{ARTICLES YOU MAY BE INTERESTED IN}

Coumarin dyes for dye-sensitized solar cells: A long-range-corrected density functional study The Journal of Chemical Physics 129, 214703 (2008); https://doi.org/10.1063/1.3025924

\section{Density-functional thermochemistry. III. The role of exact exchange}

The Journal of Chemical Physics 98, 5648 (1993); https://doi.org/10.1063/1.464913

Time-dependent density functional theory investigation of the absorption, fluorescence, and phosphorescence spectra of solvated coumarins

The Journal of Chemical Physics 125, 164324 (2006); https://doi.org/10.1063/1.2361290
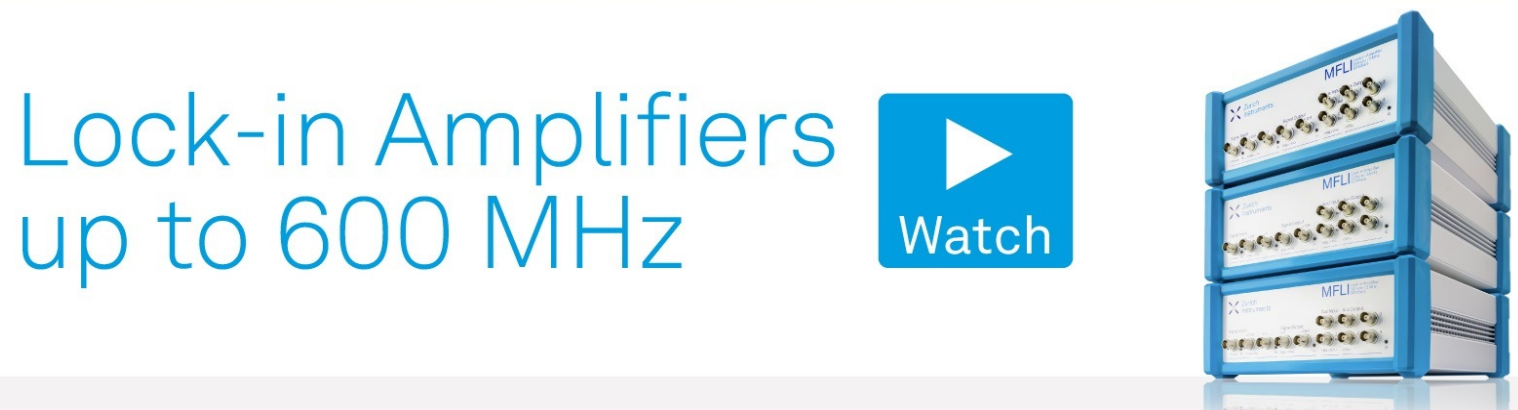


\title{
Molecular modification of coumarin dyes for more efficient dye sensitized solar cells
}

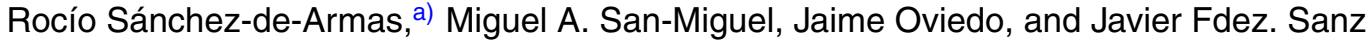 \\ Department of Physical Chemistry, University of Seville, Seville, Spain
}

(Received 1 February 2012; accepted 8 April 2012; published online 16 May 2012)

\begin{abstract}
In this work, new coumarin based dyes for dye sensitized solar cells (DSSC) have been designed by introducing several substituent groups in different positions of the NKX-2311 structure. Two types of substitutions have been considered: the introduction of three electron-donating groups $\left(-\mathrm{OH},-\mathrm{NH}_{2}\right.$, and $\left.-\mathrm{OCH}_{3}\right)$ and two different substituents with steric effect: $-\mathrm{CH}_{2}-\mathrm{CH}_{2}-\mathrm{CH}_{2}-$ and $-\mathrm{CH}_{2}-\mathrm{HC}=\mathrm{CH}-$. The electronic absorption spectra (position and width of the first band and absorption threshold) and the position of the LUMO level related to the conduction band have been used as theoretical criteria to evaluate the efficiency of the new dyes. The introduction of a $-\mathrm{NH}_{2}$ group produces a redshift of the absorption maximum position and the absorption threshold, which could improve the cell efficiency. In contrast, the introduction of $-\mathrm{CH}_{2}-\mathrm{CH}_{2}-\mathrm{CH}_{2}-$ does not modify significantly the electronic structure of NKX-2311, but it might prevent aggregation. Finally, $-\mathrm{CH}_{2}-$ $\mathrm{HC}=\mathrm{CH}-$ produces important changes both in the electronic spectrum and in the electronic structure of the dye, and it would be expected as an improvement of cell efficiency for these dyes. (C) 2012 American Institute of Physics. [http://dx.doi.org/10.1063/1.4711049]
\end{abstract}

\section{INTRODUCTION}

Coumarin dyes are one of the most promising classes of organic sensitizer ${ }^{1-4}$ for their use in dye sensitized solar cells $s^{5-7}$ and they have been systematically studied by Hara and Arakawa. ${ }^{8-14}$ They are based on the concept of the donor-acceptor $\pi$-conjugated structure (D- $\pi$-A), with the coumarin skeleton containing an amino group as the donor and a cyanoacrylic acid as the acceptor component. They are considered type-I dyes, in which electron injection occurs through an indirect mechanism: there is a photoexcitation of the dye to an excited state, followed by the electron injection from this state to the semiconductor conduction band.

A drawback related to these coumarin dyes is that they are liable to experience $\pi$-stacked aggregation on $\mathrm{TiO}_{2}$ surfaces. This type of aggregation would be advantageous for light harvesting, but it usually leads to inefficient electron injection because of intermolecular energy transfer processes between the dyes, resulting so in low cell efficiency. A strategy to prevent dye aggregation is adding coadsorbates, like deoxycholic acid and 4-tert-butylpyridine. ${ }^{13}$ Although these additives improve the electron injection, they significantly decrease dye adsorption limiting the cell performance. Another strategy that has been recently tested is to introduce a side ring in the molecule, linked to the alkene chain. This reduces the dye aggregation while keeping the adsorbed dye amount. ${ }^{14}$

Although coumarin based dyes have been extensively studied in recent years, there are not many theoretical studies about them. ${ }^{15-25}$ It is worth noting that up to date, all previous theoretical works are limited to the study of the electronic structure and the optical properties of free dyes and none of them tackles the influence of the adsorption process on the

\footnotetext{
a) Author to whom correspondence should be addressed. Electronic mail: rociosa@us.es.
}

electronic absorption spectra of these dyes. However, in several recent works, it has been demonstrated that the dye adsorption process on the semiconductor produces a reorganization of the electronic states and this fact introduces important changes in the electronic absorption spectra. ${ }^{26-28}$ Therefore, to theoretically determine the convenience of using these dyes as DSSC sensitizers, it is necessary to include the semiconductor effect explicitly in the model. In addition, the cluster size should be large enough to reproduce well the electronic structure of adsorbed dyes.

In a very recent work, several coumarin dyes have been theoretically studied with the aim of finding a relationship between some theoretically calculated properties and the efficiency of these dyes as DSSC sensitizers. ${ }^{29}$ From these results, some parameters such as the position and width of the first band in the electronic absorption spectra, the absorption threshold and the LUMO energy respect to the conduction band edge have been chosen to establish useful criteria to evaluate the efficiency of these molecules as sensitizers in DSSCs. These criteria allowed evaluating, in good agreement with experimental evidences, the efficiency order for the studied molecules.

In this work, an example of how the previously established criteria can be employed to predict the efficiency of new dyes is shown. Our aim is to design new, more efficient sensitizers, reducing economical cost and synthesis effort. We have considered the NKX-2311 dye, which presents a good efficiency as sensitizer (5.2\%) and whose electronic absorption spectrum has been previously studied in detail and has been reproduced accurately. ${ }^{29}$ The calculated absorption spectrum for the free NKX-2311 presents a main intense band at $2.30 \mathrm{eV}$ with a small shoulder at higher energies. This value is in good agreement with the experimental spectra, which presents the main band at $2.42 \mathrm{eV}$ when the spectrum 
is registered in methanol solution. ${ }^{8,9}$ The main effect that can be observed in the spectra after adsorption is a widening of the first band, while the maximum position remains almost unaltered. The main band of the adsorbed NKX-2311 spectrum appears centered at $2.23 \mathrm{eV}$ and is contributed from several excitations from HOMO orbital to different orbitals delocalized over the cluster. Nevertheless, all excitations with considerable intensity involve the LUMO +5 orbital, which is completely localized on the molecule, very similar to the LUMO of the free dye.

With the aim of designing dyes with higher efficiency than NKX-2311, several functional groups have been introduced in different positions of this molecule and its effect on the electronic structure and the absorption spectra of the dyes have been analyzed.

\section{COMPUTATIONAL METHODS AND MODELS}

Two complementary sets of calculations have been carried out for each model:

(1) DFT geometry optimizations have been done by using the Perdew-Burke-Ernzerhof (PBE) functional ${ }^{30}$ together with norm-conserving pseudopotentials ${ }^{31}$ in the fully non-local Kleinman-Bylander ${ }^{32}$ form and an auxiliary real-space grid equivalent to a plane-wave cut-off of 130 Ry. A non-standard DZP (double zeta plus polarization orbitals) basis set of Natural Atomic Orbitals (NAOs) constructed from the eigenstates of the atomic pseudopotential was used. ${ }^{33-35}$ The optical response was then computed using real time TD-DFT (time domain) simulations within the SIESTA (Spanish Initiative for Electronic Simulations with Thousands of Atoms) implementation. ${ }^{26,36-38} \mathrm{We}$ consider, as an initial state, a system in a finite electric field of $0.1 \mathrm{~V} / \AA$. Each system was allowed to evolve during 36 fs with a time-step of $1.5 \times 10^{-3}$ fs. The damping factor used in the Fourier transformation was $0.1 \mathrm{eV}$.

(2) Additionally, linear response (LR) frequency domain calculations were performed in the gas phase using the PBE functional and the 6-31G (d,p) basis set. The optical spectra were simulated by conventional TD-DFT (frequency domain) using GAUSSIAN 03. ${ }^{39}$ Over 400 singlet transitions were needed in selected calculations. Before the spectrum calculation, vibrational frequency calculations were done to confirm the stability of all the optimized geometries.

There are some advantages associated with the real-time TD-DFT formulation: all the possible excitations are generated at the same time so the spectrum is obtained in a wide range of energy; the implementation is relatively simple and although the computational cost is not cheap for small systems, it becomes competitive with traditional methods as the system size increases. However, the main disadvantage is that it is not possible to characterize the nature of the different transitions because the optical response is obtained from the analysis of the electronic dipole moment, where the information from the wavefunctions is integrated. For this rea- son, the combination with frequency domain TD-DFT is very interesting.

In a previous work, the effect of the exchange-correlation functional and the solvent has been tested for coumarin dyes, and particularly for the NKX-2311. ${ }^{29}$ Conclusions for that work have been extrapolated for the dyes considered here, which have similar structures. The frequency domain TDDFT spectra have been calculated for the free NKX-2311 using the PBE and B3LYP functionals and with and without solvent effects (PCM model). ${ }^{40,41}$ This model provides reliable absorption energies as long as no specific interactions link the solute and the solvent molecules. ${ }^{42,43}$ The position of the main peak is shifted to higher energies when passing from the PBE to the B3LYP functional, in agreement with previous published data. ${ }^{44}$ Compared to experimental spectra, $\mathrm{PBE}$ provides better results than B3LYP for NKX-2311 (although B3LYP works better when the molecule size increases). The inclusion of methanol solvation effects through a PCM model tends to reduce the excitation energies (about $0.2 \mathrm{eV}$ ), due to a stabilization of the LUMO orbital. Comparing with the previous work, we have also checked that the inclusion of longrange corrections in the functional and the use of additional diffuse base functions do not improve substantially the results for this particular dye. This is a consequence of the kind of excitation we are dealing with. As mentioned earlier, this kind of molecules work on an indirect mechanism (intramolecular excitation followed by an electron injection to the conduction band of the semiconductor). The molecules considered in this paper are not large enough to present a long-range intramolecular charge transfer (which could be underestimated by TD-DFT) and therefore, for these molecules, the photoexcited state shows a moderate charge transfer. Since for this concrete system there is not a clear choice, we have performed calculations with the PBE functional, which allows for an easier comparison between real-time and frequency calculations. An extended description of the solvent and functional influence is shown in Ref. 29.

Several substituent groups have been introduced into different sites of the NKX-2311 structure with the aim of improving its efficiency as sensitizer. The substitution sites are shown in Figure 1. Two types of substitutions have been considered. First, three different electron-donating substituents have been introduced in d position, $-\mathrm{OH},-\mathrm{NH}_{2}$, and $-\mathrm{OCH}_{3}$ and the new dyes have been labelled as D1, D2, and D3, respectively. In a previous theoretical work, it is suggested that this kind of substitution in that position could lead to an improvement of cell efficiency, due to a destabilization of the HOMO orbital. ${ }^{21}$ Second, we have introduced in the NKX2311 molecule two different substituents with steric effect:

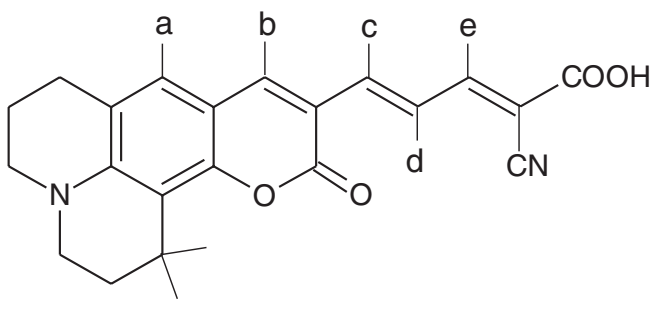

FIG. 1. Substitution sites on NKX-2311. 
TABLE I. Several substituents have been introduced in different positions of the NKX-2311 molecule to design new coumarin based dyes.

\begin{tabular}{lr}
\hline \hline Dye & Substituent and position \\
\hline $\mathrm{D} 1$ & $\mathrm{~d}=-\mathrm{OH}$ \\
$\mathrm{D} 2$ & $\mathrm{~d}=-\mathrm{NH}_{2}$ \\
$\mathrm{D} 3$ & $\mathrm{~d}=-\mathrm{OCH}_{3}$ \\
$\mathrm{AB} 1$ & $\mathrm{a}, \mathrm{b}=\uparrow$ \\
$\mathrm{BC} 1$ & $\mathrm{~b}, \mathrm{c}=\uparrow$ \\
$\mathrm{CE} 1$ & $\mathrm{c}, \mathrm{e}=\uparrow$ \\
$\mathrm{AB} 2$ & $\mathrm{a}, \mathrm{b}=\uparrow$ \\
$\mathrm{BC} 2$ & $\mathrm{~b}, \mathrm{c}=\uparrow$ \\
$\mathrm{CE} 2$ & $\mathrm{c}, \mathrm{e}=\uparrow$ \\
\hline \hline
\end{tabular}

$-\mathrm{CH}_{2}-\mathrm{CH}_{2}-\mathrm{CH}_{2}-(1)$ and $-\mathrm{CH}_{2}-\mathrm{HC}=\mathrm{CH}-(2)$. Two groups of each substituent have been introduced in the NKX-2311 in three different combinations of positions ( $a$ and $b, b$ and $\mathrm{c}$, and $\mathrm{c}$ and e) resulting in six different dyes: $\mathrm{AB} 1, \mathrm{BC} 1$, $\mathrm{CE} 1, \mathrm{AB} 2, \mathrm{BC} 2$, and $\mathrm{CE} 2$. These models are summarized in Table I.

The computational methodology has been applied to the free dyes and the same dyes adsorbed to a $\left(\mathrm{TiO}_{2}\right)_{9}$ cluster. The starting geometry for this cluster was taken from the literature. ${ }^{45,46}$ It was obtained via the geometry optimization of the originally spherical shape resulting in a compact structure with only 4-fold coordinated Ti-atoms and with one terminal Ti-O bond. In previous works, ${ }^{26,27}$ we performed real-time calculations with clusters as large as $\left(\mathrm{TiO}_{2}\right)_{38}$ and demonstrated that, even being a small cluster, $\left(\mathrm{TiO}_{2}\right)_{9}$ is the minimal size required to reproduce adequately the electronic absorption spectra of the dye- $\mathrm{TiO}_{2}$ systems. The $\left(\mathrm{TiO}_{2}\right)_{9}$ cluster is affordable from frequency domain TD-DFT calculations, which allows for a full analysis of the spectra.

These dyes present two different conformations respect to the nitrogen atom of the unsaturated rings, syn and anti, which have been reported to be almost isoenergetic. ${ }^{15}$ In this work, we have selected the syn conformer, which has also been used in previous theoretical studies. ${ }^{16,17}$ Nevertheless, it has been suggested from $a b$ initio calculations that the absorption spectra of the syn and anti isomers are almost indistinguishable for a coumarin dye. ${ }^{15}$

For D1, D2, D3, AB1, and AB2, two different conformational isomers exist. The configuration at the single bond between the coumarin moiety and the methine chain can have either an s-cis or s-trans arrangement. From previous calculations, we have seen that for NKX-2311, the $s$-cis isomer is slightly favored, due to the steric repulsion between the $\mathrm{C}=\mathrm{O}$ group in the coumarin and the $\mathrm{C}-\mathrm{N}$ group of the cyanoacrylic acid in the $s$-trans isomer. ${ }^{29}$ Nevertheless, energy differences between both isomers are insignificant (less than $0.03 \mathrm{eV}$ ). Moreover, we have checked that the calculated electronic absorption spectra of both isomers are almost identical.

In this work, for each dye we have chosen the s-cis isomer whenever possible (there is only one possible conformation for $\mathrm{BC} 1, \mathrm{CE} 1, \mathrm{BC} 2$, and $\mathrm{CE} 2)$. The dyes have been ad-
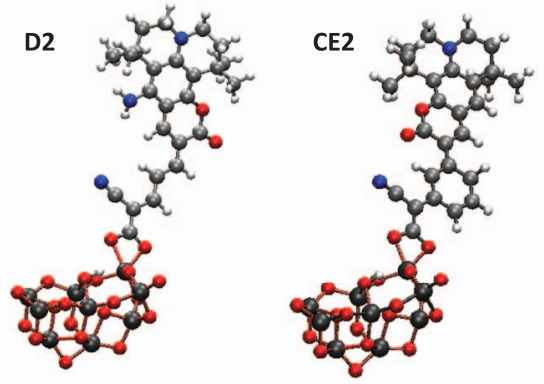

FIG. 2. Optimized geometries for the most promising dyes adsorbed on $\left(\mathrm{TiO}_{2}\right)_{9}$ : D2 (left) and CE2 (right). Dyes are adsorbed dissociated, in a bidentate chelating configuration, with the two dye oxygen atoms bound to the same surface titanium atom and the dissociated hydrogen bound to an oxygen in the center of the cluster.

sorbed dissociated, forming a bidentate chelating structure, with the two dye oxygen atoms bound to the same surface titanium atom. To keep the system electroneutral, the dissociated hydrogen atoms have been bound to an oxygen atom in the center of the cluster (Figure 2). In a previous study, we have demonstrated that this adsorption mode is the most stable one for coumarin based dyes. ${ }^{29}$ Furthermore, this chelating configuration is also found to be favorable for dyes containing carboxylate groups adsorbed on anatase surfaces. ${ }^{47}$

To predict the new dyes efficiency, we have made the use of criteria published previously. ${ }^{29}$ On comparing two dyes with similar structures, the light harvesting would be more efficient for the dye with a lower absorption threshold and a wider first band in the electronic absorption spectrum. Furthermore, the electron injection would be more efficient for that dye with the higher excited state related to the semiconductor conduction band edge. Therefore, we will use three criteria to evaluate an improvement of the efficiency of the new dyes: the electronic absorption spectra (position and width of the first band and absorption threshold) and the position of the LUMO level related to the conduction band.

\section{RESULTS}

\section{A. Electron-donating substituents in d position}

The simulated absorption spectra from real-time TDDFT calculations for adsorbed D1, D2, and D3 dyes are shown in Figure 3 (left). Adsorbed NKX-2311 absorption spectrum has been included for comparison. The D1 and D3 spectra show a profile very similar to the NKX-2311 one; they present a main intense band with a small shoulder at higher energies and the main peak maximum almost in the same position. On the other hand, for D2, the introduction of a $-\mathrm{NH}_{2}$ group in d position produces a redshift of about $0.1 \mathrm{eV}$ on the absorption maximum position and also a redshift on the absorption threshold compared to NKX-2311, extending absorption into the visible region.

For adsorbed D1 and D3, both excitations and orbital schemes are very similar to those for NKX-2311, which was described in detail in a previous work. ${ }^{29}$ In general, for these three adsorbed systems, molecular orbitals are either completely localized on the cluster or fully localized on the dye molecule. However, for the adsorbed D2, more 

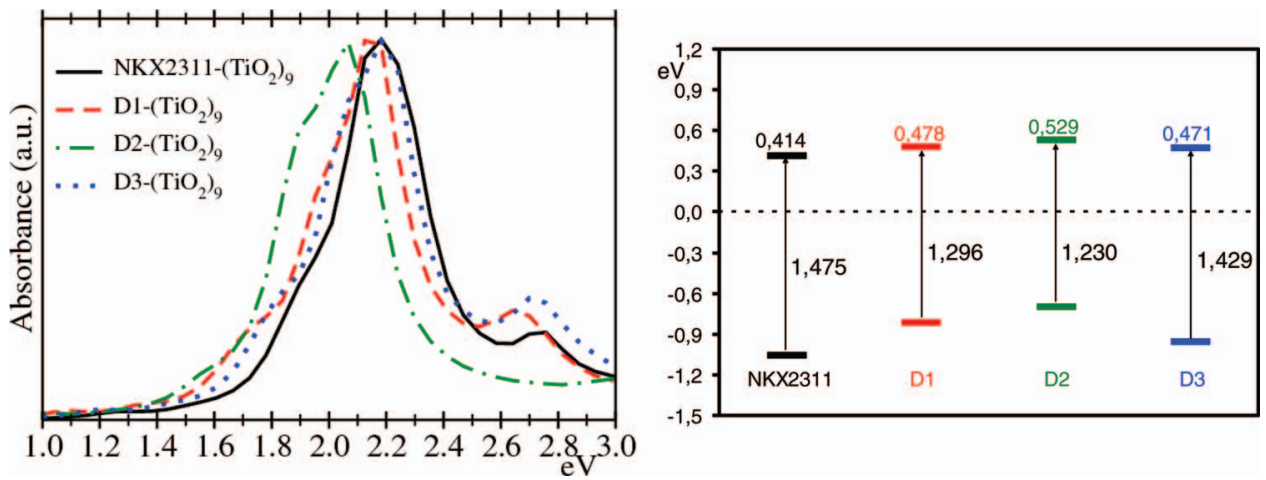

FIG. 3. Simulated real-time TD-DFT spectra (left) and HOMO and LUMO* energies (right) for adsorbed NKX-2311, D1, D2, and D3. In the energy scheme, the orbital energies have been shifted to set the zero energy to the lower edge of the semiconductor conduction band.

mixing between cluster and dye orbitals is observed and every virtual orbital shows some contribution from the cluster. The LUMO+7 orbital corresponds to the LUMO of the free dye and it is delocalized over the whole system (Figure 4), while for NKX-2311, D1, and D3, this orbital has no contribution from the cluster. From here on, for each system, we will use the name LUMO* (or $\mathrm{L}^{*}$ ) to refer to the orbital of the adsorbed system, which is more similar to the LUMO orbital of the free dye.

The adsorbed D2 spectrum presents a main wide band centered at $2 \mathrm{eV}$ with a small shoulder at lower energy (centered at $1.7 \mathrm{eV}$ ). This shoulder corresponds to an excitation from the HOMO orbital, fully localized on the molecule, to LUMO+13 (Figure 4), an orbital fully localized on the cluster (Table II). However, in this band, there are minor contributions from the LUMO+7 (LUMO*) and LUMO+8 orbitals, both delocalized over the whole system. The main band includes several excitations from HOMO orbital to different virtual orbitals; some of them delocalized over the cluster and others delocalized over the whole system, such as LUMO*.

To complete the analysis, the HOMO and LUMO* energies for the four adsorbed dyes have been represented in Figure 3 (right). In this representation, the LUMO orbital energy has been set to zero. The LUMO orbital is, for every system, an orbital fully localized on the cluster and very similar to the same orbital of the free cluster. In consequence, the zero value corresponds to the lower edge of the semiconductor conduction band.

For all modified dyes, the gap between HOMO and LUMO is smaller than for the unsubstituted dye. The dif-

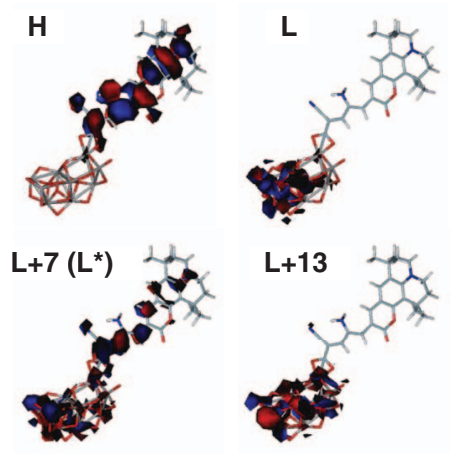

FIG. 4. Occupied and virtual molecular orbitals for adsorbed D2. ference with NKX-2311 HOMO-LUMO energy gap is rather small for D3 (less than $0.05 \mathrm{eV}$ ) and bigger for D2 $(0.25 \mathrm{eV})$, reflecting so an important redshift for the absorption maximum for D2. The decrease in the HOMO-LUMO energy gap is due to a destabilization of the HOMO orbital, which is maximum for D2. Moreover, for the three dyes, LUMO* orbital is less stable than for NKX-2311. This destabilization is small for D1 and D3 $(0.06 \mathrm{eV}$ ) but it is more important for D2 (about $0.1 \mathrm{eV}$ ).

The higher the LUMO* orbital is located in the semiconductor conduction band, the more efficient the electron injection is. Regarding this criterion, we might only expect a noticeable improvement of the efficiency for the D2 dye. For this dye, LUMO* is located higher related to the lower edge of the $\mathrm{TiO}_{2}$ conduction band, so it is located in a zone where the effective density of states is higher. This makes the electron injection easier and it also explains that there is more mixing between the cluster and dye orbitals for this dye.

\section{B. Substituents with steric effect}

We have introduced into the NKX-2311 molecule the groups $-\mathrm{CH}_{2}-\mathrm{CH}_{2}-\mathrm{CH}_{2}-$ (1) and $-\mathrm{CH}_{2}-\mathrm{HC}=\mathrm{CH}-$ (2).

TABLE II. Assignments of electronic excitations for adsorbed D2 dye. Only selected transitions with enough oscillator strength around the main peak are included. (H stands for HOMO and L for LUMO).

\begin{tabular}{|c|c|c|}
\hline $\begin{array}{l}\text { Transition energy } \\
(\mathrm{eV})\end{array}$ & $\begin{array}{l}\text { Oscillator } \\
\text { strength }\end{array}$ & Wavefunction \\
\hline \multicolumn{3}{|r|}{ Shoulder } \\
\hline \multirow[t]{3}{*}{1.67} & 0.0755 & $\mathrm{H} \rightarrow \mathrm{L}+13(0.66), \mathrm{H} \rightarrow \mathrm{L}+12(0.15)$ \\
\hline & & $\mathrm{H} \rightarrow \mathrm{L}+7(-0.10) \mathrm{H} \rightarrow \mathrm{L}+8(-0.10)$ \\
\hline & \multicolumn{2}{|c|}{ Main band } \\
\hline 1.82 & 0.0636 & $\mathrm{H} \rightarrow \mathrm{L}+15(0.68)$ \\
\hline 1.94 & 0.1154 & $\mathrm{H} \rightarrow \mathrm{L}+17(0.65), \mathrm{H} \rightarrow \mathrm{L}+16(-0.12)$ \\
\hline \multirow[t]{7}{*}{2.06} & 0.9507 & $\mathrm{H} \rightarrow \mathrm{L}+7$ (0.24), $\mathrm{H} \rightarrow \mathrm{L}+8(0.24)$ \\
\hline & & $\mathrm{H} \rightarrow \mathrm{L}+17(-0.23), \mathrm{H} \rightarrow \mathrm{L}+6(-0.18$ \\
\hline & & $\mathrm{H} \rightarrow \mathrm{L}+13(0.16), \mathrm{H} \rightarrow \mathrm{L}+15(0.14)$ \\
\hline & & $\mathrm{H} \rightarrow \mathrm{L}+19(-0.14), \mathrm{H} \rightarrow \mathrm{L}+21(0.13)$ \\
\hline & & $\mathrm{H} \rightarrow \mathrm{L}+5$ (0.12), $\mathrm{H} \rightarrow \mathrm{L}+9(0.11)$ \\
\hline & & $\mathrm{H} \rightarrow \mathrm{L}+16(-0.11), \mathrm{H} \rightarrow \mathrm{L}+20(0.11)$ \\
\hline & & $\mathrm{H} \rightarrow \mathrm{L}+18(-0.10)$ \\
\hline
\end{tabular}



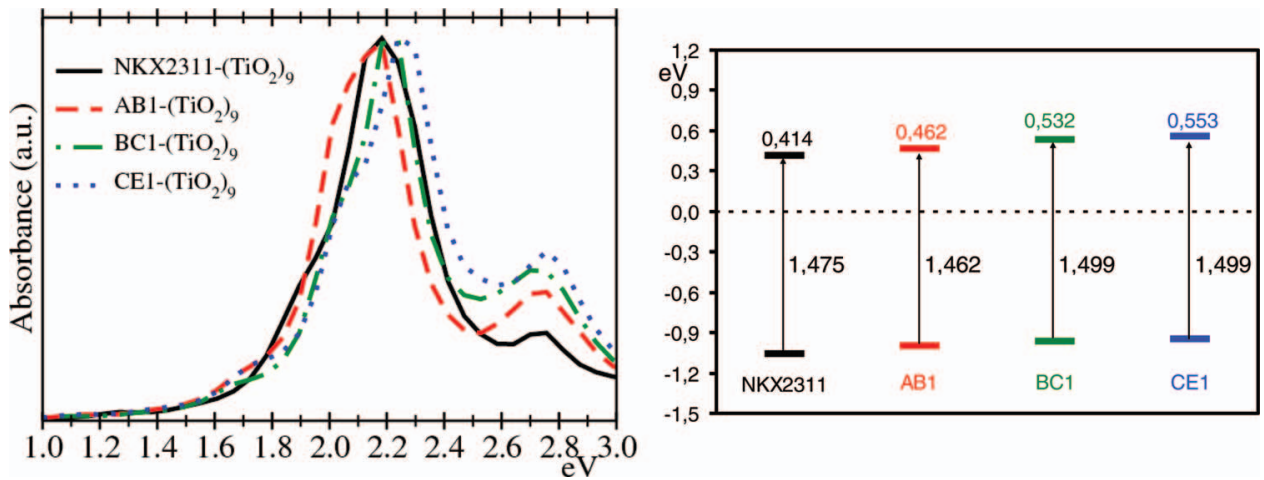

FIG. 5. Simulated real-time TD-DFT spectra (left) and HOMO and LUMO* energies (right) for adsorbed NKX-2311, AB1, BC1, and CE1. In the energy scheme, the orbital energies have been shifted to set the zero energy to the lower edge of the semiconductor conduction band.

These groups bind to two different $\mathrm{C}$ atoms of NKX-2311, leading to the formation of a new ring in the molecule.

The introduction of the first substituent $\left(-\mathrm{CH}_{2}-\mathrm{CH}_{2}-\right.$ $\mathrm{CH}_{2}-$ ) does not produce important changes in the electronic structure or the electronic spectrum of NKX-2311. The position of the absorption maximum remains almost unaltered for $\mathrm{AB} 1$ and $\mathrm{BC} 1$ and is slightly blue-shifted for CE1 (Figure 5 left).

The main contribution to the first band and the orbital scheme are also very similar to those for NKX-2311. AB1 presents a HOMO-LUMO* energy gap slightly smaller than NKX-2311 and both HOMO and LUMO* are slightly more unstable than those for adsorbed NKX-2311 ones (Figure 5 right). However, differences are too small to predict an improvement of the cell efficiency. For BC1 and CE1, the LUMO destabilization is more important $(0.11-0.13 \mathrm{eV})$, which would favor electronic injection, but it entails an increase of the energy gap between the HOMO and LUMO* orbitals, which is disadvantageous for the light harvesting process. Therefore, the criteria do not permit to predict an improvement of the cell efficiency using these new sensitizers. Nevertheless, the introduction of a new ring in the molecule could prevent dye aggregation, which would lead to an improvement of the cell efficiency. Since our model contains only one dye molecule, we cannot study aggregation effects in this work.

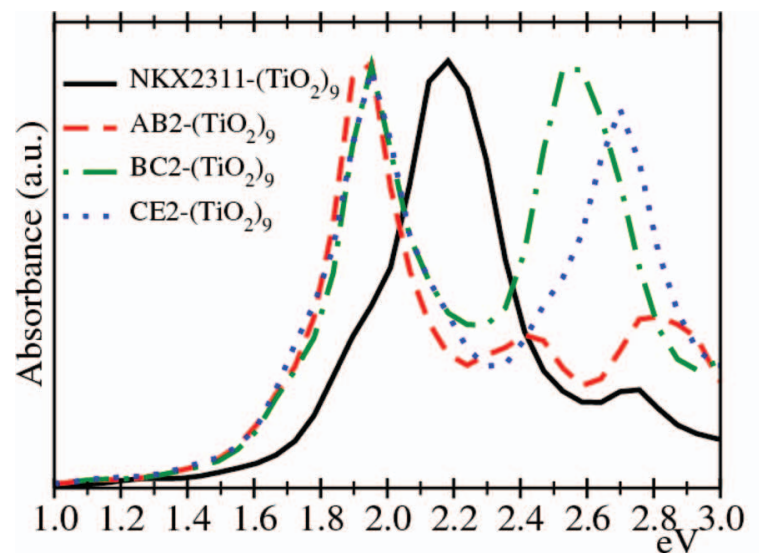

On the other hand, the introduction of the second substituent $\left(-\mathrm{CH}_{2}-\mathrm{HC}=\mathrm{CH}-\right)$ produces important changes in the electronic absorption spectra: the position of the absorption maximum redshifts about $0.2 \mathrm{eV}$ for the three new dyes (AB2, $\mathrm{BC} 2$, and CE2). For the three dyes, the spectrum presents a main band with a very similar shape; it is centred at 1.95 $\mathrm{eV}$ and has a small shoulder at lower energies (Figure 6 left). While introducing a new ring in the molecule, this substituent introduces also a new double bond, which expands the $\pi$ conjugation in the dye, responsible for the redshift and the absorption widening in the visible region, desirable for sunlight harvesting.

Nevertheless, at higher energies there are some differences between the spectra. In adsorbed NKX-2311 spectra, there is a small shoulder of the main band at $2.8 \mathrm{eV}$. This shoulder is slightly more intense for adsorbed AB2 and it becomes an intense well-resolved peak for BC2 and CE2.

The assignments of the more intense electronic excitations for adsorbed CE2 dye spectrum are shown in Table III. The spectrum shows a main band centered at about $1.9 \mathrm{eV}$, which presents a small shoulder at lower energy centered at $1.6 \mathrm{eV}$. The main contribution to this shoulder corresponds to an excitation from the HOMO orbital, fully localized on the molecule, to the LUMO +8 , an orbital delocalized over the whole cluster, but with an appreciable molecule contribution. The main band includes several excitations from the HOMO

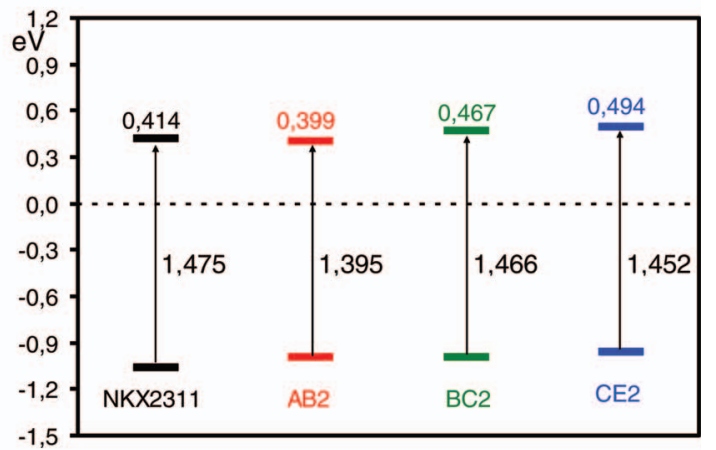

FIG. 6. Simulated real-time TD-DFT spectra (left) and HOMO and LUMO* energies (right) for adsorbed NKX-2311, AB2, BC2, and CE2. In the energy scheme, the orbital energies have been shifted to set the zero energy to the lower edge of the semiconductor conduction band. 
TABLE III. Assignments of electronic excitations for adsorbed CE2 dye. Only selected transitions with enough oscillator strength around the main peak are included. (H stands for HOMO and L for LUMO).

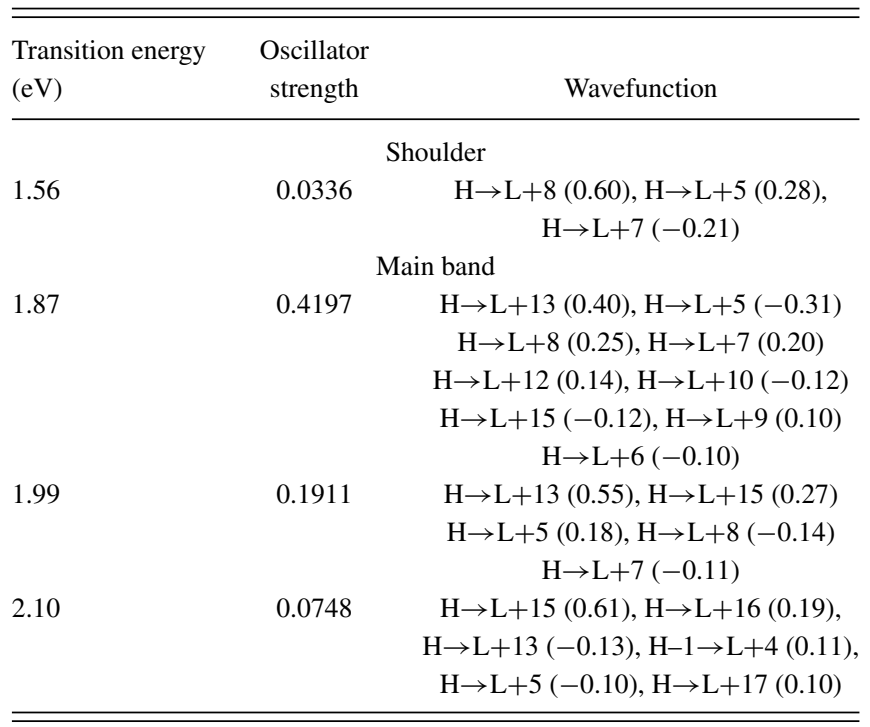

orbital to different orbitals fully localized in the cluster, without any molecular contribution (LUMO+13, LUMO+15). Nevertheless, all excitations with noticeable intensity have a relatively important contribution of the excitation from the $\mathrm{HOMO}$ to the LUMO +5 orbital. The latter corresponds to the LUMO of the free dye (LUMO*), although it has a considerable contribution from the cluster. Representative frontier occupied and virtual molecular orbitals of adsorbed CE2 are shown in Figure 7.

The HOMO-LUMO* energy gap for AB2, BC2, and CE2 is smaller than for NKX-2311 (Figure 6 right). The expansion of the $\pi$ conjugation in the dye produces a destabilization of the HOMO orbital. For BC2 and CE2, the LUMO* orbital is also less stable than for NKX-2311, which makes the electron injection process easier for these two new dyes. Combining the different criteria, we predict that introducing a $-\mathrm{CH}_{2}-\mathrm{HC}=\mathrm{CH}-$ group in the molecule could lead to an improvement in the cell efficiency, being the most promising dye CE2, because it combines the bigger redshift of the spectrum maximum position and absorption threshold with a more important HOMO destabilization and with a higher LUMO* orbital related to the lower edge of the semiconductor conduction band.

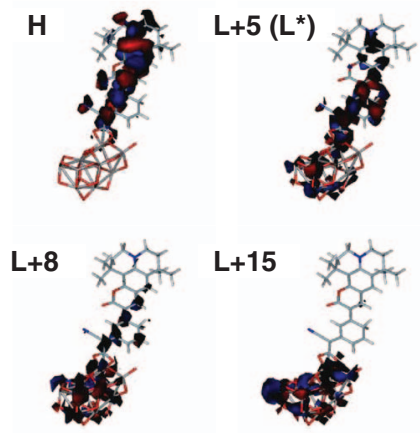

FIG. 7. Occupied and virtual molecular orbitals for adsorbed CE2.

\section{CONCLUSIONS}

In summary, in this work, we show how we can employ the electronic absorption spectra (position and width of the first band and absorption threshold) and the position of the LUMO level related to the conduction band as criteria to evaluate the efficiency of new coumarin based dyes. We have introduced several functional groups in the NKX-2311 molecule with the aim of improving its efficiency as DSSC sensitizer. The introduction of a $-\mathrm{NH}_{2}$ group in $\mathrm{d}$ position could lead to an efficiency improvement due to an important HOMO destabilization and a slightly LUMO destabilization, which produces a redshift of the absorption maximum position and the absorption threshold. The introduction of $-\mathrm{CH}_{2}-\mathrm{CH}_{2}-\mathrm{CH}_{2}-$ does not introduce significant changes in the electronic structure of NKX-2311, but it could prevent the formation of aggregates. Finally, $-\mathrm{CH}_{2}-\mathrm{HC}=\mathrm{CH}-$ produces important changes both in the electronic spectrum and in the electronic structure of the dye, and we might expect an improvement of the cell efficiency for AB2, BC2, and CE2. In particular, the CE2 would be the most promising dye, because it combines a redshift in the spectrum with a bigger HOMO destabilization and a bigger energy gap between LUMO* and the edge of the semiconductor conduction band.

\section{ACKNOWLEDGMENTS}

This work was funded by the Spanish Ministerio de Ciencia e Innovación, MICINN (Project Nos. MAT2008-4918 and CSD2008-0023). R.S.A. thanks the Junta de Andalucía for a predoctoral grant (P08-FQM-3661 and EXC/2005/FQM1126). Part of the calculations has been carried out at the Andalusia Supercomputing Center, Servicio de Supercomputación del Centro Informático Científico de Andalucía (C.I.C.A).

${ }^{1}$ A. Mishra, M. K. R. Fischer, and P. Bäulere, Angew. Chem. Int. Ed. 48, 2474 (2009).

${ }^{2}$ Y. Ooyama and Y. Harima, Eur. J. Org. Chem. 18, 2903 (2009).

${ }^{3}$ Z. Chen, F. Li, and C. Huang, Curr. Org. Chem. 11, 1241 (2007).

${ }^{4}$ D. Jacquemin, J. Preat, E. A. Perpete, and C. Adamo, Int. J. Quant. Chem. 110, 2121 (2010).

${ }^{5}$ M. Grätzel, Nature 414, 338 (2001).

${ }^{6}$ W. R. Duncan and O. V. Prezhdo, Annu. Rev. Phys. Chem. 58, 143 (2007).

${ }^{7}$ Z. Ning, Y. Fu, and H. Tian, Energy Environ. 3, 1170 (2010).

${ }^{8}$ K. Hara, K. Sayama, Y. Ohga, A. Shinpo, S. Suga, and H. Arakawa, Chem. Comm. 6, 569 (2001).

${ }^{9}$ K. Hara, T. Sato, R. Katoh, A. Furube, Y. Ohga, A. Shinpo, S. Suga, K. Sayama, H. Sugihara, and H. Arakawa, J. Phys. Chem. B 107, 597 (2003).

${ }^{10}$ K. Hara, Y. Tachibana, Y. Ohga, A. Shinpo, S. Suga, K. Sayama, H. Sugihara, and H. Arakawa, Sol. Energy Mater. Sol. Cells 77, 89 (2003).

${ }^{11}$ K. Hara, M. Kurashige, Y. Dan-Oh, C. Kasada, A. Shinpo, S. Suga, K. Sayama, and H. Arakawa, New J. Chem. 27, 783 (2003).

${ }^{12}$ Z. Wang, Y. Cui, Y. Dan-Oh, C. Kasada, A. Shinpo, and K. Hara, J. Phys. Chem. C 112, 17011 (2008).

${ }^{13}$ K. Hara, Y. Dan-Oh, C. Kasada, Y. Ohga, A. Shinpo, S. Suga, K. Sayama, and H. Arakawa, Langmuir 20, 4205 (2004).

${ }^{14}$ Z. Wang, K. Hara, Y. Dan-oh, C. Kasada, A. Shinpo, S. Suga, H. Arakawa, and H. Sugihara, J. Phys. Chem. B 109, 3907 (2005).

${ }^{15}$ R. Improta, V. Barone, and F. Santoro, Angew. Chem. Int. Ed. 46, 405 (2007).

${ }^{16}$ Y. Kurashige, T. Nakajima, S. Kurashige, and K. Hirao, J. Phys. Chem. A 111, 5544 (2007).

${ }^{17}$ B. M. Wong and J. G. Cordaro, J. Chem. Phys. 129, 21403 (2008). 
${ }^{18}$ T. Stein, L. Kronik, and R. Baer, J. Chem. Phys. 131, 244119 (2009).

${ }^{19}$ M. P. Balanay, S. Kim, M. J. Lee, S. H. Lee, and D. H. Kim, Bull. Korean Chem. Soc. 30, 2077 (2009).

${ }^{20}$ K. Deuk Seo, H. Min Song, M. Jun Lee, M. Pastore, C. Anselmi, F. De Angelis, M. K. Nazeeruddin, M. Gräetzel, and H. Kyu Kim, Dyes Pigm. 90, 304 (2011).

${ }^{21}$ X. Zhang, J. Zhang, and Y. Xia, J. Photochem. Photobiol. A: Chem. 194, 167 (2008).

${ }^{22}$ D. Jacquemin, E. A. Perpète, G. Scalmani, M. J. Frisch, X. Assfeld, I. Ciofini, and C. Adamo, J. Chem. Phys. 125, 164324 (2006).

${ }^{23}$ I. Georgieva, N. Trendafilova, A. Aquino, and H. Lischka, J. Phys. Chem. A 109, 11860 (2005).

${ }^{24}$ R. J. Cave and E. W. Castner, Jr., J. Phys. Chem. 106, 12117 (2002).

${ }^{25}$ M. Sulpizi, P. Carloni, J. Hutter, and U. Rothlisberger, Phys. Chem. Chem. Phys. 5, 4798 (2003).

${ }^{26}$ R. Sánchez-de-Armas, J. Oviedo, M. A. San Miguel, P. Ordejón, M. Pruneda, and J. F. Sanz, J. Chem. Theory Comput. 6, 2856 (2010).

${ }^{27}$ R. Sánchez-de-Armas, M. A. San-Miguel, J. Oviedo, A. Márquez, and J. F. Sanz, Phys. Chem. Chem. Phys. 13, 1506 (2011).

${ }^{28}$ R. Sánchez-de-Armas, J. Oviedo, M. A. San-Miguel, and J. F. Sanz, J. Phys. Chem. C 115, 11293 (2011).

${ }^{29}$ R. Sánchez-de-Armas, M. A. San-Miguel, J. Oviedo, and J. F. Sanz, Phys. Chem. Chem. Phys. 14, 225 (2012).

${ }^{30}$ J. P. Perdew and K. Burke, Phys. Rev. Let. 77, 3865 (1996).

${ }^{31}$ N. Troullier and J. L. Martins, Phys. Rev. B 43, 1993 (1991).

${ }^{32}$ L. Kleinman and D. M. Bylander, Phys. Rev. Lett. 48, 1425 (1982).
${ }^{33}$ E. Artacho, D. Sánchez-Portal, P. Ordejón, A. García, and J. M. Soler, Phys. Status Solidi B 215, 809 (1999).

${ }^{34}$ J. Junquera, O. Paz, D. Sanchez-Portal, and E. Artacho, Phys. Rev. B 64, 235111 (2001).

${ }^{35}$ E. Anglada, J. Soler, J. Junquera, and E. Artacho, Phys. Rev. B 66, 205101 (2002).

${ }^{36}$ J. M. Soler, E. Artacho, J. D. Gale, A. García, J. Junquera, P. Ordejón, and D. Sánchez-Portal, J. Phys. Condens. Matter. 14, 2745 (2002).

${ }^{37}$ D. Sánchez-Portal, P. Ordejón, E. Artacho, and J. M. Soler, Int. J. Quant. Chem. 67, 453 (1997).

${ }^{38}$ A. Tsolakidis, D. Sánchez-Portal, and R. M. Martin, Phys. Rev. B 66, 235416 (2002).

${ }^{39}$ M. J. Frisch, G. W. Trucks, H. B. Schlegel et al., GAUSSIAN 03, Gaussian, Inc., Wallingford, CT, 2003.

${ }^{40}$ M. Cossi and V. Barone, J. Chem. Phys. 112, 2427 (2000).

${ }^{41}$ M. Cossi and V. Barone, J. Chem. Phys. 115, 4708 (2001).

${ }^{42}$ R. Improta, G. Scalmani, M. J. Frisch, and V. Barone, J. Chem. Phys. 127, 074504 (2007).

${ }^{43}$ J. P. Cerón-Carrasco, A. Ripoche, F. Odobel, and D. Jacquemin, Dyes Pigm. 92, 1144 (2012)

${ }^{44}$ M. Pastore, S. Fantacci, and F. De Angelis, J. Phys. Chem. 114, 22742 (2010).

${ }^{45}$ Z. Qu and G. Kroes, J. Phys. Chem. B 110, 8998 (2006).

${ }^{46}$ S. Hamad, C. R. A. Catlow, S. M. Woodley, S. Lago, and J. A. Mejías, J. Phys. Chem. B 109, 15741 (2005).

${ }^{47}$ X. Gong, A. Selloni, and A. Vittadini, J. Phys. Chem. 110, 2804 (2006). 